\title{
Clinical implications of perivascular fat stranding surrounding spontaneous isolated superior mesenteric artery dissection on computed tomography
}

\author{
ZHENGWU TAN $^{1,2}$, QIANNA JIN ${ }^{1,2}$, WENLIANG FAN ${ }^{1,2}$, PING HAN ${ }^{1,2}$ and XIN LI ${ }^{1,2}$ \\ ${ }^{1}$ Department of Radiology, Union Hospital, Tongji Medical College, Huazhong University of Science and Technology, \\ Wuhan, Hubei 430022; ${ }^{2}$ Hubei Province Key Laboratory of Molecular Imaging, Wuhan, Hubei 430022, P.R. China
}

Received April 23, 2020; Accepted October 9, 2020

DOI: $10.3892 /$ etm.2020.9466

\begin{abstract}
Patients with spontaneous isolated superior mesenteric artery (SMA) dissection (SISMAD) usually present with acute or chronic abdominal pain and are admitted to the emergency or digestive diseases department to undergo auxiliary examinations, typically abdominal plain CT or contrast-enhanced CT (CECT). Plain CT is the most crucial examination in emergency radiology. An enlarged SMA diameter and perivascular fat stranding (PFS) on plain $\mathrm{CT}$, though non-specific, may be the only indications for SISMAD. These results may be easily overlooked and the diagnosis of SISMAD may be missed. However, PFS around the SMA on CT may be the only indicator of the possible presence of SISMAD, particularly during the early stage when there are no massive changes in the vascular wall. The present study aimed to determine whether PFS surrounding the SMA on CT may help with the diagnosis of SISMAD by indicating the requirement for further examination. The data of 161 consecutive patients with SMA dissection who underwent abdominal CECT or underwent SMA CT angiography (CTA) after abdominal plain CT between February 2015 and February 2018 were retrospectively reviewed. SMA diameter, classification, PFS, complications, comorbidities and treatments were analyzed. The results demonstrated that SISMAD with PFS was significantly associated with admission type (emergency), clinical manifestations (abdominal
\end{abstract}

Correspondence to: Dr Xin Li, Department of Radiology, Union Hospital, Tongji Medical College, Huazhong University of Science and Technology, 1277 Jiefang Avenue, Wuhan, Hubei 430022, P.R. China

E-mail: 1xwsry2014@163.com

Abbreviations: PFS, perivascular fat stranding; SISMAD, spontaneous isolated superior mesenteric artery dissection; CECT, contrast-enhanced CT; CTA, CT angiography

Key words: superior mesenteric artery, dissection, acute abdomen, emergency, perivascular fat stranding pain), diagnostic modality and dissection subtype. On plain CT, PFS surrounding the SMA may be a marker for SISMAD, particularly in the emergency setting, and indicates the requirement for CTA examination.

\section{Introduction}

Arterial dissection is defined as the cleavage of the arterial wall by an intramural hematoma (1). Isolated visceral arterial dissection, i.e., dissection that occurs in the absence of aortic dissection, has been reported to involve the celiac artery and renal arteries; however, the most frequent site of isolated dissection is the superior mesenteric artery (SMA). SMA dissection was first described by Bauersfeld (2-4) in 1947 and may be categorized into: i) spontaneous isolated SMA dissection (SISMAD) and ii) SMA dissection combined with aortic dissection. The latter type is more common and is caused by the extension of an aortic dissection flap into the SMA (5). By contrast, SISMAD is a rare but potentially fatal condition.

Prior to 2012, no more than 270 cases of SISMAD were reported in the PubMed database (3). The development of advanced imaging technologies, such as multi-detector CT (CT), has likely led to an increase in the detection of SISMAD (3). Since 2016, >622 cases of SISMAD have been reported (6). CT imaging with intravenous contrast administration is able to clearly determine the location and extent of the dissection in patients with SISMAD (6). However, in a considerable subset of patients, SISMAD presents with atypical clinical symptoms (e.g., acute or chronic abdominal pain) or is entirely asymptomatic. Therefore, in numerous cases, plain CT is the first imaging examination that such patients undergo (3,7-9). Furthermore, plain CT remains an important examination in emergency departments and hospitals with limited medical resources $(6,10)$. However, the characteristic imaging findings of SISMAD, namely an intimal flap and a mural thrombus, may not be visible on plain CT $(8,11,12)$.

Suzuki et al (13) reported that increased perivascular fat density around the SMA is a sign of arterial dissection or thrombosis. The normal perivascular fat around the SMA appears as homogeneous low attenuation signals on CT and on CT angiography (CTA), the normal SMA walls have a 


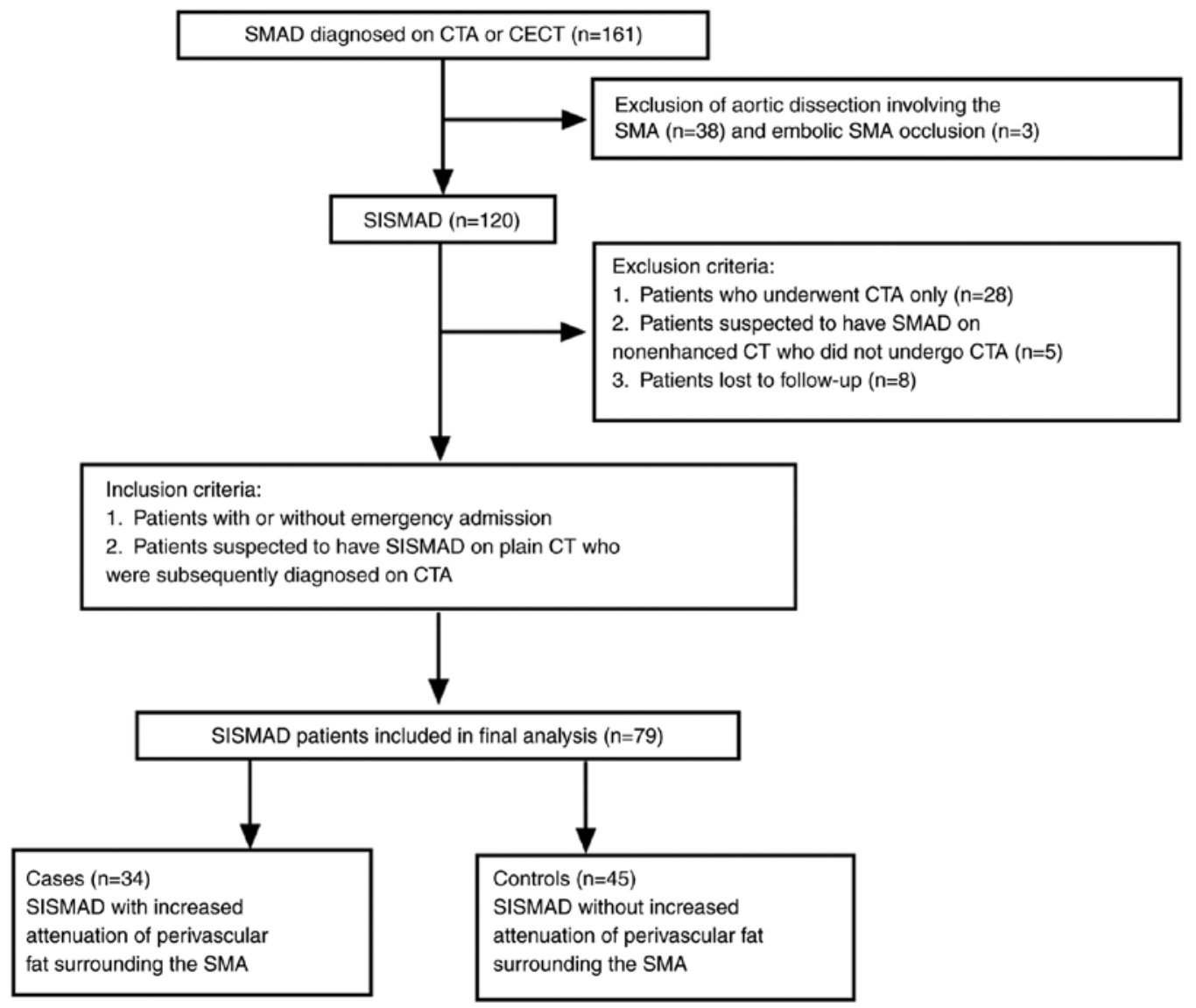

Figure 1. Flowchart of patient selection. SMA, superior mesenteric artery; SMAD, SMA dissection; CTA, CT angiography; CECT, contrast-enhanced CT; SISMAD, spontaneous isolated SMAD.

distinct interface with the perivascular fat (14). Perivascular fat stranding (PFS) is the CT finding of abnormally increased attenuation in fat tissue. PFS is a manifestation of edema, inflammation and/or neoplastic infiltration and was first detected on abdominopelvic CT (15).

The purpose of the present study was to determine whether the detection of PFS on plain CT scans may serve as a useful indicator of the requirement for further examination with contrast-enhanced CT (CECT) or CTA to diagnose or rule out SISMAD.

\section{Materials and methods}

Patient population. The medical and imaging data of consecutive patients who were diagnosed with SISMAD on abdominal CECT or SMACTA at our hospital between February 2015 and February 2018 were reviewed. Certain patients initially underwent non-enhanced abdominal CT, which was suggestive of SMA dissection; hence, these patients subsequently underwent CTA for the diagnosis of SISMAD. Patients who were admitted to the emergency department, as well as those who were not, were included in the study. A flow chart of patient selection and the clinical inclusion and exclusion criteria for patients with SISMAD are presented in Fig. 1. The demographic and clinical characteristics of the patients with SISMAD are summarized in Table I. The present retrospective registry study was approved by the institutional review board of our hospital.
Image acquisition and analysis. Due to the retrospective nature of the present study, images were acquired using a variety of CT scanners (Somatom Definition from Siemens AG; Aquilion One from Toshiba; and Discovery CT750 HD from Cytiva). The scans included both CECT and CTA following non-enhanced abdominal CT according to our institution's protocol. The scanning parameters were as follows: i) for CTA: $100 \mathrm{kV}$; auto mAs; slice thickness, 0.625-1.0 mm; ii) non-enhanced CT or CECT: 100 or $120 \mathrm{kV}$; auto mAs; and slice thickness, 1.25-2.0 mm. For vessel reconstruction, the axial imaging data were transferred to an Advanced Workstation for post-processing (Extended Brilliance Workspace, EBW). The image of the SMA was reformatted using volume rendering, maximum intensity projection, multiplanar reconstruction and curved planar reconstruction.

The diagnosis of SISMAD was confirmed when one of the following signs was seen in the SMA: i) Intimal flap and contrast enhancement within the false lumen; and ii) a crescent-shaped area along the wall of the SMA indicating no contrast enhancement $(2,16,17)$. All images were jointly reviewed by two radiologists (Examiner 1 and Examiner 2, with 5 and 20 years of experience in abdominal radiology, respectively) who were blinded to the patients' history. Any disagreements were resolved through consensus. The presence or absence of PFS was ascertained using reformatted multiplanar plain abdominal CT and precontrast CT scans that were free of any motion artifacts. The case group was defined as patients with PFS, while the control group was defined as patients without 
Table I. Demographic and clinical characteristics of patients with SISMAD ( $n=79)$.

\begin{tabular}{|c|c|c|c|}
\hline Characteristics & Cases $(n=34)$ & Controls $(n=45)$ & P-value \\
\hline Mean age (years) & $55.06 \pm 6.958$ & $55.98 \pm 9.538$ & 0.136 \\
\hline Male sex & $31(91.2)$ & $42(93.3)$ & 1.000 \\
\hline Type of admission & & & 0.030 \\
\hline Emergency & $22(64.7)$ & $18(40.0)$ & \\
\hline Non-emergency & $12(35.3)$ & $27(60.0)$ & \\
\hline Clinical presentation & & & 0.024 \\
\hline Abdominal pain & $29(85.3)$ & $28(62.2)$ & \\
\hline No abdominal pain ${ }^{\mathrm{a}}$ & $5(14.7)$ & $17(37.7)$ & \\
\hline Comorbidities & & & 0.888 \\
\hline Hypertension & $13(38.2)$ & $21(46.7)$ & \\
\hline Smoking (current or ex-smoker) & $12(35.3)$ & $17(37.8)$ & \\
\hline Alcohol consumption (current or ex-drinker) & $10(29.4)$ & $17(37.8)$ & \\
\hline Diabetes mellitus & $2(5.9)$ & $1(2.2)$ & \\
\hline Hyperlipidemia & $3(8.8)$ & $2(4.4)$ & \\
\hline Liver cirrhosis & $1(2.9)$ & $3(6.7)$ & \\
\hline Gastric ulcer & $1(2.9)$ & $1(2.2)$ & \\
\hline Malignancy & & & 0.906 \\
\hline Hepatocellular carcinoma & $1(2.9)$ & $1(2.2)$ & \\
\hline Colorectal carcinoma & $1(2.9)$ & $1(2.2)$ & \\
\hline Stomach cancer & $2(5.9)$ & $1(2.2)$ & \\
\hline Diagnostic modality & & & 0.017 \\
\hline Contrast-enhanced CT & $16(47.1)$ & $33(73.3)$ & \\
\hline CT angiography & $18(52.9)$ & $12(26.7)$ & \\
\hline Time interval between plain CT and CT angiography (days) & 3.5 & 4.6 & \\
\hline
\end{tabular}

Patients had more than one comorbidity. ${ }^{\mathrm{N}}$ No abdominal pain: Patients with SISMAD who were admitted to hospital not because of abdominal pain but because of other conditions, including gastrointestinal or liver tumor, obstructive jaundice, acute pancreatitis, gastrointestinal hemorrhage, health examination, neurogenic bladder, hematuria and infective endocarditis. Values are expressed as n (\%) unless otherwise specified. Groups: Cases, patients with SISMAD and PFS; patients with SISMAD without PFS. SISMAD, spontaneous isolated superior mesenteric artery dissection; PFS, perivascular fat stranding.

PFS. Axial, coronal, sagittal, as well as multiplanar reconstruction images obtained using abdominal CECT or CTA were reviewed for the diagnosis of SISMAD. Each of these images was analyzed for the following categories: Diameters of the affected segment and the unaffected proximal segment, and the classification, complications, comorbidities and management of SISMAD. The SISMAD classifications described by previous studies $(3,9,10,12,16,18,19)$ were used to divide SISMAD into types I to IV. In addition, each type was further divided, if possible, into the following subtypes: Subtype L, long segmental dissection (i.e., dissection extending distally into the ileocolic or distal ileal artery); subtype S, significant stenosis of the true lumen (i.e., $>80 \%$ ); and subtype LS, a combination of subtypes $\mathrm{L}$ and $\mathrm{S}$ (Fig. 2).

Statistical analysis. Continuous variables are expressed as the mean \pm standard deviation. Categorical variables are presented as numbers and percentages. Continuous variables were compared between the case and control groups by using the Student's t-test or the Mann-Whitney U-test. Categorical variables were compared between the two groups by using the
Pearson chi-square test, Fisher's exact test or Wilcoxon test. All statistical analyses were performed using SPSS (version 19; IBM, Corp.). $\mathrm{P}<0.05$ was considered to indicate a statistically significant difference.

\section{Results}

General information. A total of 161 patients (137 males, 24 females; mean age, 54.7 years) were diagnosed with SMA dissection on CTA or CECT. Of these patients, 38 patients with combined aortic and SMA dissection and 3 patients with embolic SMA occlusion were excluded. In addition, 28 patients with SISMAD who underwent only CTA examination, 5 patients who were suspected to have SMA dissection according to the non-enhanced CT findings but did not undergo CTA examination and 8 patients who were lost to follow-up were excluded. Thus, 79 patients with SISMAD were included in the final analysis (Fig. 1).

Of these 79 patients, $34(43.0 \%)$ patients with PFS [including $31(91.2 \%)$ males] were included in the case group, while $45(57 \%)$ patients without PFS [including $42(93.3 \%)$ 


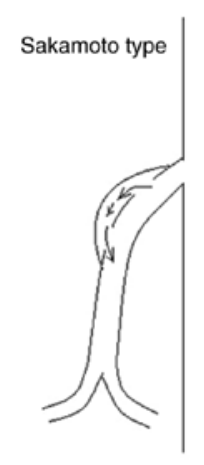

Type I

Subtype

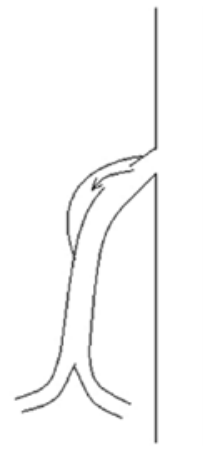

Type II

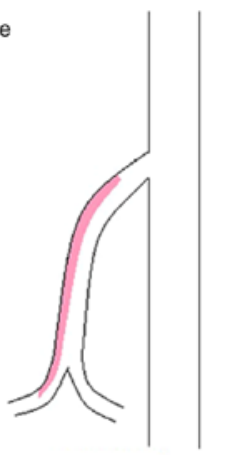

Type L
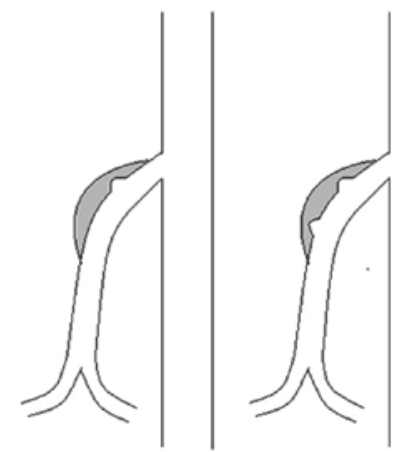

Type III

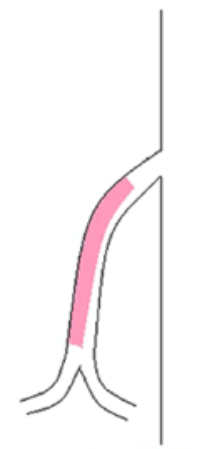

Type S

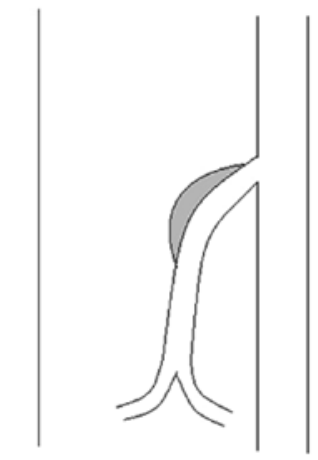

Type IV

Figure 2. Classification of SISMAD. Type I, patent false lumen with both entry and re-entry; type II, patent false lumen without re-entry; type III, thrombosed false lumen with ulcer-like projection; type IV, completely or partially thrombosed false lumen. Each SISMAD type was additionally classified into the following subtypes if possible: Subtype L, long dissection segment (i.e., dissection extending distally to the ileocolic or distal ileal artery); subtype S, significant true lumen stenosis (>80\%); and subtype LS, combination of subtypes L and S. SISMAD, spontaneous isolated superior mesenteric artery dissection.

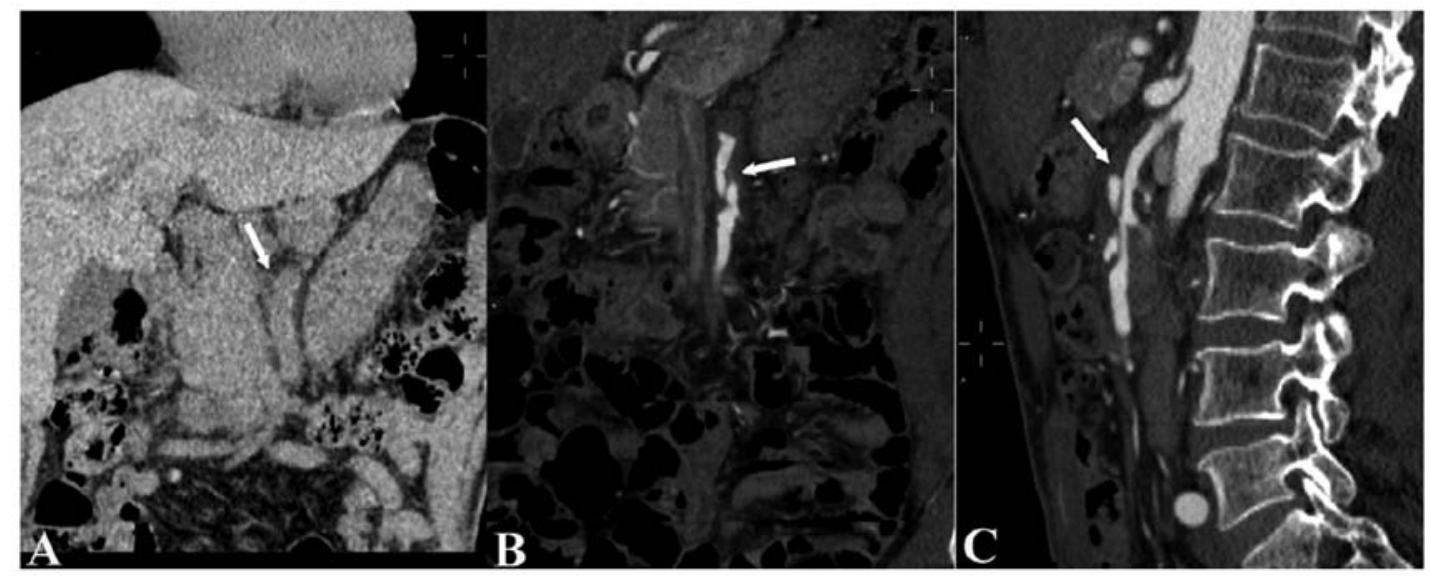

Figure 3. A 50-year-old male patient was admitted to the hospital with abdominal pain and underwent non-enhanced CT examination of the upper abdomen in the emergency radiology department. (A) Non-enhanced CT revealed perivascular fat stranding surrounding the SMA (arrow) without enlargement of the SMA diameter and other definite abnormalities in the upper abdomen. (B and C) CT angiography performed $4 \mathrm{~h}$ later demonstrates a partially thrombosed false lumen (arrows) without either a long dissection segment or true lumen stenosis $>80 \%$, which was classified as type III spontaneous isolated SMA dissection. SMA, superior mesenteric artery.

males] were assigned to the control group. The mean age of the patients in the case and control groups was $55.06 \pm 6.958$ years (range, 44-69 years) and 55.98 \pm 9.538 years (range, 42-82 years), respectively. Age and gender did not significantly differ between the case and control groups ( $>>0.05$; Table I).

Patient characteristics. The percentage of patients requiring emergency admission was significantly higher in the case group than in the control group (64.7 vs. $40.0 \%, \mathrm{P}=0.030)$ and the incidence of PFS was significantly higher in the emergency group than in the non-emergency group (55.5 vs. $30.8 \%, \mathrm{P}=0.030$ ). The clinical presentation of SISMAD ranged from asymptomatic to acute peritonitis. The most common symptom was abdominal pain, which was present in $29(85.3 \%)$ patients in the case group and $28(62.2 \%)$ patients in the control group $(\mathrm{P}=0.024$; Table I). The proportion of patients admitted to the emergency and non-emergency departments did not significantly differ 
Table II. Clinical presentation of the patients with SISMAD $(\mathrm{n}=79)$.

\begin{tabular}{lccr}
\hline Setting/abdominal pain & Total & Cases & Controls \\
\hline Emergency & $37(92.5)^{\mathrm{a}}$ & $21(56.8)$ & 16 \\
$\quad$ Abdominal pain & 3 & $1(33.3)$ & 2 \\
No abdominal pain & & $8(40.0)$ & 12 \\
Non-emergency & $20(51.3)$ & $4(21.1)$ & 15 \\
$\quad$ Abdominal pain & 19 & 0.301 \\
No abdominal pain & b & & \\
\hline
\end{tabular}

${ }^{\mathrm{a}} \mathrm{P}<0.001$ vs. no abdominal pain. ${ }^{\mathrm{b}}$ No abdominal pain: Patients with SISMAD who were admitted to the hospital not because of abdominal pain but because of other conditions, including gastrointestinal or liver tumor, obstructive jaundice, acute pancreatitis, gastrointestinal hemorrhage, health examination, neurogenic bladder, hematuria or infective endocarditis. Values are expressed as n (\%). Groups: Cases, patients with SISMAD and PFS; patients with SISMAD without PFS SISMAD, spontaneous isolated superior mesenteric artery dissection; PFS, perivascular fat stranding.
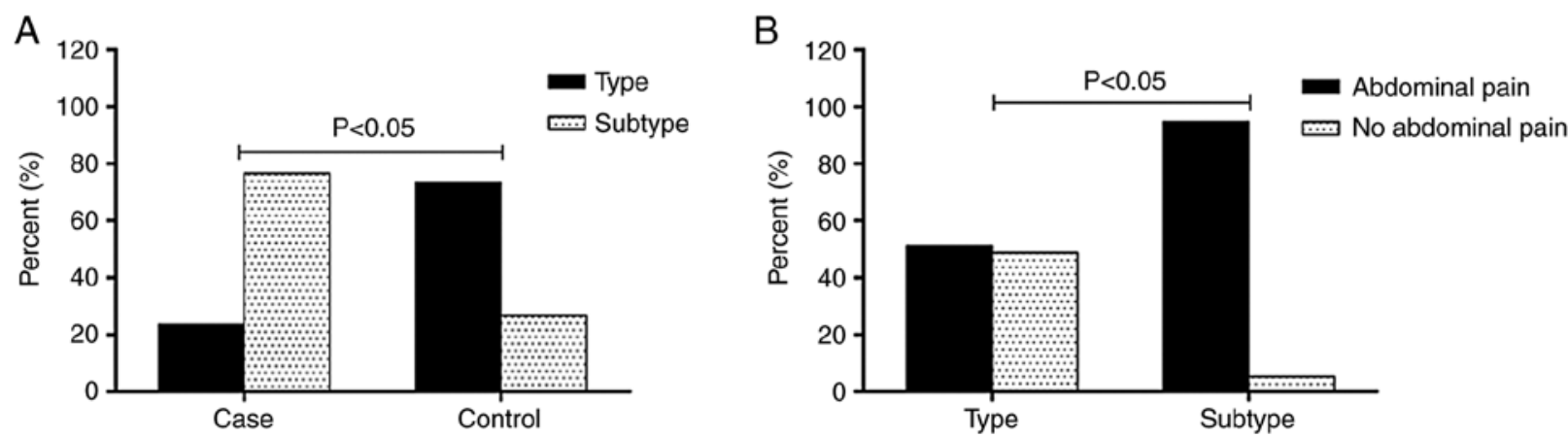

Figure 4. (A) Classification of SISMAD in the case and control groups. Subtypes were assigned significantly more frequently in patients with SISMAD and PFS than in patients without PFS [76.5\% (26/34) vs. 26.7\% (12/45), P<0.001]. (B) Clinical presentation according to SISMAD classification. Patients with assigned subtypes of dissection were associated with abdominal pain significantly more frequently than those that were not able to be assigned subtypes [94.7\% (36/38) vs. 51.2\% (21/41); P<0.001]. Groups: Cases, patients with SISMAD and PFS; patients with SISMAD without PFS. SISMAD, spontaneous isolated superior mesenteric artery dissection; PFS, perivascular fat stranding.

between the two study groups $(\mathrm{P}=0.856$ and $\mathrm{P}=0.301$, respectively; Table II). However, the proportion of patients with abdominal and no abdominal pain differed significantly between the emergency and non-emergency groups $(\mathrm{P}<0.001$; Table II). SISMAD was diagnosed using CTA in 18 patients in the case group (Fig. 3) and 12 patients in the control group $(\mathrm{P}=0.017$; Table I). The mean time interval between the initial non-enhanced CT and the subsequent CTA examination was slightly shorter in the case group than in the control group, but the difference was not significant.

Comorbidities. All 79 patients had various coexisting medical conditions, including malignancies (Table I). The most common comorbidity was hypertension $(n=34)$, followed by smoking $(n=29)$, alcohol intake $(n=27)$ and hyperlipidemia $(n=5)$, although the percentages of these comorbidities were low. All asymptomatic patients were diagnosed through the incidental detection of SMA dissection on CT during an examination for other conditions, including gastrointestinal or liver tumor, obstructive jaundice, acute pancreatitis, gastrointestinal hemorrhage, general health examination, neurogenic bladder, hematuria or infective endocarditis. There were no significant differences in coexisting medical conditions and malignancies between the two groups.
SISMAD classification. As presented in Table III, the diameters of the affected and unaffected segments of the SMA differed significantly from each other in both the case group $(\mathrm{P}<0.001)$ and the control group $(\mathrm{P}<0.001)$; however, these diameters did not differ between the case and control groups (affected segment, $\mathrm{P}=0.363$; unaffected segment, $\mathrm{P}=0.307$ ). According to the CT findings, SISMAD was classified as follows: Type I, $24.05 \%$ of patients; type II, $8.86 \%$ of patients; type III, $33.97 \%$ of patients; and type IV, $29.11 \%$ of patients. The proportions of these types did not significantly differ between the case and control groups $(\mathrm{P}=0.058)$. Type III and I was the most common type in the case and control group, respectively (Table III). A total of 38 patients had a subtype L, S or LS dissection and these subtypes were significantly more common in the case group than in the control group [26 (76.47\%) vs. $12(26.27 \%)$ patients; $\mathrm{P}<0.001$; Fig. 4A]. None of the type I lesions could be classified into subtypes L, S or LS. Of the 3 type II lesions, 1 was classified as subtype II-L and 2 as subtype II-S. Among the 22 type III lesions, there were 15 subtype III-L, 2 subtype III-S and 5 subtype III-LS lesions. The 13 type IV lesions were divided into 3 subtype IV-L, 1 subtype IV-S and 9 subtype IV-LS lesions. Subtypes I and IV were significantly less common in the case group than in the control group, while subtypes III-S 
Table III. CT findings and management of the patients with SISMAD ( $\mathrm{n}=79)$.

\begin{tabular}{|c|c|c|c|}
\hline Characteristics & Cases $(n=34)$ & Controls $(n=45)$ & P-value \\
\hline \multicolumn{4}{|l|}{ Diameter (mm) } \\
\hline Affected segment & $9.79 \pm 1.452$ & $10.24 \pm 2.577$ & 0.363 \\
\hline Unaffected proximal segment & $5.88 \pm 0.844$ & $5.67 \pm 0.977$ & 0.307 \\
\hline Classification $^{\mathrm{a}}$ & & & 0.058 \\
\hline Type I & $4(11.8)$ & $15(33.3)$ & \\
\hline Type II & $0(0)$ & $4(8.9)$ & \\
\hline Type II-L & $1(2.9)$ & $0(0)$ & \\
\hline Type II-S & $2(5.9)$ & $0(0)$ & \\
\hline Type III & $2(5.9)$ & $6(13.3)$ & \\
\hline Type III-L & $11(32.4)$ & $4(8.9)$ & \\
\hline Type III-S & $2(5.9)$ & $0(0)$ & \\
\hline Type III-LS & $2(5.9)$ & $3(6.7)$ & \\
\hline Type IV & $2(5.9)$ & $8(17.8)$ & \\
\hline Type IV-L & $3(8.8)$ & $0(0)$ & \\
\hline Type IV-S & $0(0)$ & $1(2.2)$ & \\
\hline Type IV-LS & $5(14.7)$ & $4(8.9)$ & \\
\hline \multicolumn{4}{|l|}{ Concomitant findings } \\
\hline Celiac trunk dissection & $5(14.7)$ & $10(22.2)$ & 0.399 \\
\hline Renal artery dissection & $1(2.9)$ & $0(0)$ & 0.887 \\
\hline Intestinal necrosis & $1(2.9)$ & $4(8.9)$ & 0.543 \\
\hline Treatment & & & 0.126 \\
\hline Conservative & $27(79.4)$ & $29(64.4)$ & \\
\hline Endovascular & $5(14.7)$ & $9(0.2)$ & \\
\hline Interventional thrombolysis & $1(2.9)$ & $3(6.7)$ & \\
\hline Surgery & $1(2.9)$ & $4(8.9)$ & \\
\hline
\end{tabular}

${ }^{a}$ There were no type I-L, type I-S, type I-LS or type II-LS lesions in the case or control groups. The classification was according to Fig. 2. Values are expressed as $n(\%)$ or the mean \pm standard deviation. Groups: Cases, patients with SISMAD and PFS; patients with SISMAD without PFS. SISMAD, spontaneous isolated superior mesenteric artery dissection; PFS, perivascular fat stranding.

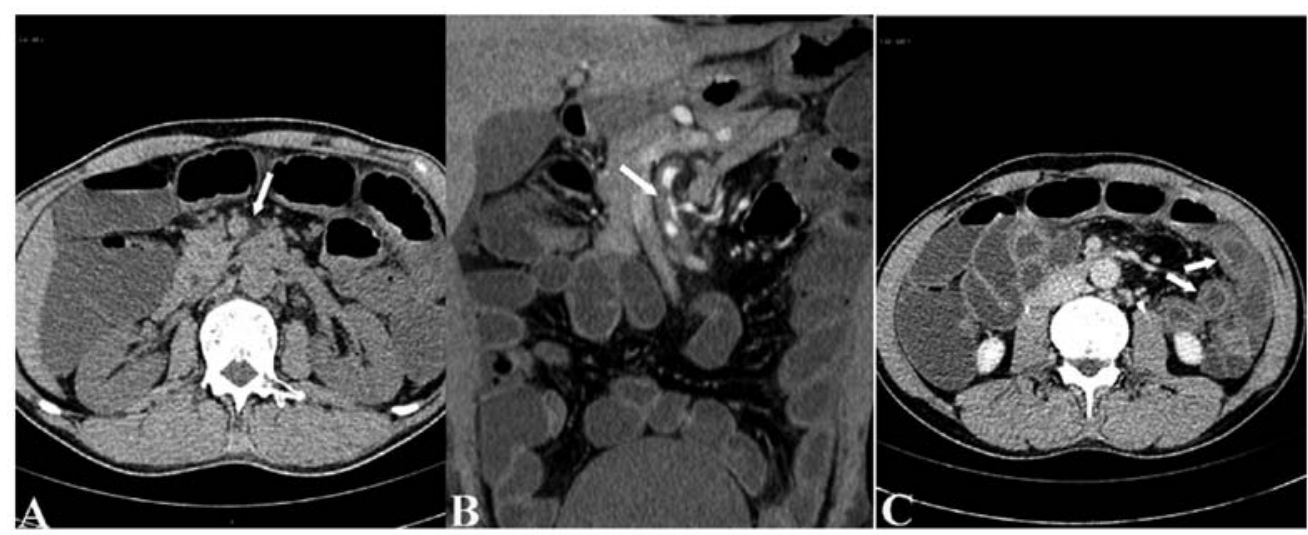

Figure 5. A 50-year-old male was admitted to the hospital with abdominal pain and hematochezia and underwent plain CT examination of the total abdomen at the emergency radiology department. (A) Non-enhanced CT revealed perivascular fat stranding surrounding the SMA (arrow) without enlargement of the SMA diameter and other definite abnormalities in the abdomen. (B) Contrast-enhanced CT performed 1 day later demonstrated a partially thrombosed false lumen (arrows) with severe stenosis of the true lumen (>80\%), which was classified as type III-S spontaneous isolated SMA dissection. (C) In addition, thickened, weak and enhancing intestinal walls were seen, suggesting ischemia or edema of the walls (arrows). SMA, superior mesenteric artery.

(Fig. 5) and IV-L were more common in the case group than in the control group. Of the 34 patients in the case group,
29 (85.3\%) were symptomatic. Abdominal pain was significantly more common in patients with subtype L, S and LS 
lesions (particularly in the case of type III and IV lesions) than in patients without subtypes (Fig. 4B).

Complications and outcomes. In the overall study cohort, SISMAD was accompanied by celiac trunk dissection in 15 patients and with renal artery dissection in 1 patient. In addition, 5 patients developed intestinal necrosis as a complication. The incidence of these complications did not significantly differ between the case and control groups. In total, 56 patients underwent conservative treatment, including antiplatelet and anticoagulation therapy (27 cases and 29 controls). Surgical intervention was eventually required in 5 of these 56 patients due to suspicion of severe intestinal ischemia or necrosis on CT scan and blood test results (Table III).

\section{Discussion}

The present study demonstrated that SISMAD with PFS was significantly associated with admission type (emergency), clinical manifestations (abdominal pain), diagnostic modality and dissection subtype. Thus, PFS is a potential cause of abdominal pain in patients with SISMAD and the detection of PFS on plain CT scans may be suggestive of a diagnosis of SISMAD and indicates the requirement for further investigation.

To the best of our knowledge, ultrasound examination has a limited value in the diagnosis of SMA disease due to the influence of intestinal gas. Furthermore, emergency magnetic resonance imaging is difficult and expensive. Therefore, CTA is currently the most sensitive and reliable method for diagnosing SISMAD. At our different hospitals, CTA is the diagnostic test of choice for patients suspected to have SISMAD, while CECT is the most commonly used test when SISMAD is diagnosed incidentally in China $(5,6,17)$. However, in clinical practice, numerous patients cannot directly undergo CTA and rather undergo plain CT first. This is because of the influence of various factors such as atypical clinical manifestations and a low clinical suspicion for this condition. Therefore, abdominal plain CT is the most important examination in routine radiological work-up, particularly in the emergency radiological work-up for abdominal pain.

The specific etiology of SISMAD is unclear and multiple risk factors have been suggested to be related to this arteriopathy, including connective tissue diseases, cystic medial necrosis, arterial wall dysfunction involving atherosclerosis, fibromuscular dysplasia, vasculitis, tobacco use, atherosclerosis, segmental arterial mediolysis, alcohol abuse, obesity, heavy weight lifting, history of smoking or hypertension, pregnancy and hemodynamic forces caused by the convex curvature of the SMA $(1,3,6,18,20-25)$. Patients with SISMAD were predominantly males with an average age of 55 years and this may be related to male smoking and drinking behaviors $(6,17)$. Perhaps, SISMAD can be prevented by measures such as cessation of smoking and drinking alcohol and keeping good dietary habits $(6,22)$.

The imaging characteristics of SISMAD include an intimal flap, a mural thrombus and an intramural hematoma, which may not be visible on plain CT (11). Other CT findings suggestive of dissection include an enlarged SMA diameter and PFS around the SMA. Although non-specific, PFS is a critical clue for SMA dissection, particularly when no aneurysmal dilatation or definitive findings are present on the initial plain CT $(1,8,12,13,15,22,26)$. Therefore, identifying the sign of PFS on plain CT is helpful to not miss the diagnosis of SISMAD. The rate of PFS among all the SISMAD patients in the present study was only $43 \%$ (34/79); however, among the $30(38.0 \%)$ patients who initially underwent non-enhanced CT prior to CTA examination, PFS was detected in $60 \%(18 / 30)$. In clinical practice, most patients with SISMAD do not initially have indications for CECT or CTA due to the atypical symptoms of SISMAD. Therefore, the possibility of SISMAD is usually not considered in these patients, who typically undergo plain CT. However, as this disease may cause fatal intestinal ischemia or necrosis, a timely and accurate diagnosis is important. Suzuki et al (13) reported on the case of a patient with acute abdominal pain in whom the initial CT scan indicated PFS around the SMA without any definite abnormalities in the SMA; a second examination performed 1 month later confirmed SISMAD. Patients with SISMAD cannot be screened out based on clinical symptoms alone; therefore, indirect signs such as PFS that may be sensitively detected on plain CT may be useful to indicate the requirement for further CTA or CECT examination to diagnose or rule out SISMAD.

PFS around the SMA is a pathological condition (15,26-28) and may be caused by edema or inflammation of the fat. Endovascular injury, thinning of the vascular wall and increased permeability cause the inflammatory cells in the vessels to migrate to the perivascular fat, which leads to the augmented interaction of local adipocytes and inflammatory cells within the periadventitial adipose tissue. This interaction changes in response to endovascular injury (29) and induces periadventitial fat inflammation. The cause of abdominal pain in SISMAD is unclear. Certain studies reported that the pain is related to mesenteric ischemia caused by stenosis of the true lumen or to the dissection itself $(3,7,10,13,30)$. In the present study, the proportion of patients with abdominal pain was higher in the case group than in the control group, possibly because perivascular inflammation stimulates visceral neuroplexuses, causing abdominal pain $(1,13,31)$. This is also consistent with abdominal pain being more common in patients requiring emergency treatment in the case group. Inflammation beyond the intima has been described in patients with acute coronary syndrome (14) and arterial dissection $(32,33)$. Therefore, PFS may also represent the acute phase of intimal tearing during dissection $(31,34)$. Of all patients with abdominal pain, $64.9 \%$ (37/57) were initially admitted to the emergency department, of which $56.8 \%$ (21/37) were in the case group. Patients with SISMAD and PFS mainly complained of abdominal pain at the time of presentation and were therefore admitted to the emergency department.

Early classifications of SISMAD were based mainly on morphological appearance, and disease severity was incorporated in later classifications $(3,7-9,12,20,35)$. Despite significant advances in our understanding and diagnosis of this condition, no consensus has emerged regarding which classification and management strategy are optimal. The ideal SISMAD classification should reflect both its morphological features and the severity of the disease. In addition, it should be as simple as possible. The more detailed classification systems established by Yoo et al (3), Sakamoto et al (9) and Luan and Li (12), which are all based on morphological appearance, clinical symptoms 
and management, were adopted in the present study. The results indicated that none of the dissection types significantly differed between the case and control groups. This may be attributable to a type of selection bias, as only patients who were admitted to hospital were studied; asymptomatic patients may not be admitted to a hospital and the diagnosis may be missed. In addition, the present study was limited to a single institution. However, the prevalence of subtypes III-L and IV-L was high in patients with PFS and type-III lesions were more common than other lesion types in the case group, which is consistent with previous studies (35). The proportion of patients with abdominal pain was significantly greater in the case of lesions that were able to be assigned a subtype than in lesions that were not. It may be speculated that PFS leads to clinical symptoms owing to the involvement of a longer segment of the SMA and the aggravation of stenosis $(12,18)$. Patients with type-III or -IV lesions are likely to present with acute abdominal symptoms (6). It appears logical that longer dissections and/or more severe true lumen stenosis would cause more inflammation and thus pain.

In the present study, abnormalities of the celiac trunk and renal arteries were observed in 16 patients. This suggests that radiological evaluation of SISMAD should not be limited to the SMA and attempts should be made to evaluate the morphological characteristics of the rest of the abdominal arterial system. A total of 5 patients developed intestinal ischemia, only 1 of whom was in the case group; the remaining patients with severe stenosis in the case group did not develop intestinal ischemia. It may be postulated that collateral flow from the celiac artery and inferior mesenteric artery or patent branches of the proximal SMA may have a critical role in preventing the development of bowel infarction during the early stage of dissection (7). The goals of SISMAD treatment are symptom relief, as well as prevention of intestinal necrosis and SMA rupture. Therefore, the management of patients with SISMAD tends to depend on the presence or absence of symptoms $(7,8,10,12,19)$.

The present study had certain limitations. First, the number of cases with PFS was small, which may be associated with the pain endurance of certain patients, who may not have noticed their own symptoms, as well as with the retrospective study design, due to which selection bias cannot be excluded. Furthermore, motion artifacts were common distractors while evaluating images for the presence of fat stranding. However, the images were evaluated by two readers to estimate the inter-observer consistency in detecting the sign of PFS and reduce this error. In addition, patients were included in the study based on whether there was SISMAD and patients with embolic occlusion of the SMA who may have had PFS were excluded. As another limitation, SISMAD was not able to be distinguished from an intramural hematoma of the SMA, as the density of hematoma and dissection are not distinctly different on plain CT (13). Therefore, it is possible that certain cases of SISMAD were missed. In addition, symptom severity was not assessed in detail in patients with mild abdominal pain who were admitted to the emergency department in accordance with China's national regulations.

In conclusion, although PFS surrounding the SMA on $\mathrm{CT}$ has been reported in the literature, to our knowledge, the frequency and clinical implications of PFS surrounding SISMAD on CT have not been previously described. To the best of our knowledge, the present study was the first to focus on PFS in patients with SISMAD. The present results indicated that PFS was significantly associated with abdominal pain, type of admission (emergency) and SISMAD classification. The results suggest that when PFS surrounding the SMA is detected on plain CT in a patient presenting with abdominal pain, particularly in an emergency setting, further CTA examination should be performed to diagnose or rule out SISMAD. It is esteemed that this CT marker may help in the timely detection and treatment of SISMAD and thereby prevent the development of life-threatening complications, such as intestinal necrosis.

\section{Acknowledgements}

Not applicable.

\section{Funding}

The present study was supported by the National Natural Science Foundation of China (grant no. 81701673) and the Natural Science Foundation of Hubei Province.

\section{Availability of data and materials}

The datasets used and analyzed during the present study are available from the corresponding author on reasonable request.

\section{Authors' contributions}

XL designed the study. XL, QJ and ZT collected the data. $\mathrm{XL}, \mathrm{ZT}, \mathrm{QJ}$ and $\mathrm{PH}$ reviewed the imaging data. ZT and WF analyzed the data, reviewed the charts and interpreted the data. ZT and QJ performed a literature search and selected the studies to be included. XL and ZT wrote the manuscript. PH and QJ edited the manuscript. All authors read and approved the final version of the manuscript.

\section{Ethics approval and consent to participate}

The present retrospective registry study was approved by the institutional review board of our hospital.

\section{Patient consent for publication}

Not applicable.

\section{Competing interests}

The authors declare that they have no competing interests.

\section{References}

1. D'Ambrosio N, Friedman B, Siegel D, Katz D, Newatia A and Hines J: Spontaneous isolated dissection of the celiac artery: CT findings in adults. AJR Am J Roentgenol 188: W506-W511, 2007.

2. Satokawa H, Takase S, Seto Y, Yokoyama H, Gotoh M, Kogure M, Midorikawa H, Saito T and Maehara K: Management strategy of isolated spontaneous dissection of the superior mesenteric artery. Ann Vasc Dis 7: 232-238, 2014.

3. Yoo J, Lee JB, Park HJ, Lee ES, Park SB, Kim YS and Choi BI: Classification of spontaneous isolated superior mesenteric artery dissection: Correlation with multi detector CT features and clinical presentation. Abdom Radiol (NY) 43: 3157-3165, 2018. 
4. Bauersfeld SR: Dissecting aneurysm of the aorta; a presentation of 15 cases and a review of the recent literature. Ann Intern Med 26: 873-889, 1947.

5. Ghodasara N, Liddell R, Fishman EK and Johnson PT: High-value multidetector CT angiography of the superior mesenteric artery: What emergency medicine physicians and interventional radiologists need to know. Radiographics 39: 559-577, 2019.

6. Luan JY, Guan X, Li X, Wang CM, Li TR, Zhang L and Han JT: Isolated superior mesenteric artery dissection in China. J Vasc Surg 63: 530-536, 2016.

7. Kim HK, Jung HK, Cho J, Lee JM and Huh S: Clinical and radiologic course of symptomatic spontaneous isolated dissection of the superior mesenteric artery treated with conservative management. J Vasc Surg 59: 465-472, 2014.

8. Park WJ and Seo JW: Follow-up with computed tomography after spontaneous isolated dissection of the splanchnic artery. Clin Imaging 52: 1-7, 2018.

9. Sakamoto I, Ogawa Y, Sueyoshi E, Fukui K, Murakami T and Uetani M: Imaging appearances and management of isolated spontaneous dissection of the superior mesenteric artery. Eur J Radiol 64: 103-110, 2007.

10. Zerbib P, Perot C, Lambert M, Seblini M, Pruvot FR and Chambon JP: Management of isolated spontaneous dissection of superior mesenteric artery. Langenbecks Arch Surg 395: 437-443, 2010

11. Schwartz SA, Taljanovic MS, Smyth S, O'Brien MJ and Rogers LF: CT findings of rupture, impending rupture, and contained rupture of abdominal aortic aneurysms. AJR Am J Roentgenol 188: W57-W62, 2007.

12. Luan JY and Li X: Computed tomography imaging features and classification of isolated dissection of the superior mesenteric artery. Eur J Vasc Endovasc Surg 46: 232-235, 2013.

13. Suzuki S, Furui S, Kohtake H, Sakamoto T, Yamasaki M, Furukawa A, Murata K and Takei R: Isolated dissection of the superior mesenteric artery: CT findings in six cases. Abdom Imaging 29: 153-157, 2004.

14. Hedgire S, Baliyan V, Zucker EJ, Bittner DO, Staziaki PV Takx RAP, Scholtz JE, Meyersohn N, Hoffmann U and Ghoshhajra B: Perivascular epicardial fat stranding at coronary CT angiography: A marker of acute plaque rupture and spontaneous coronary artery dissection. Radiology 287: 808-815, 2018.

15. Thornton E, Mendiratta-Lala M, Siewert B and Eisenberg RL: Patterns of fat stranding. AJR Am J Roentgenol 197: W1-W14, 2011.

16. Li DL, He YY, Alkalei AM, Chen XD, Jin W, Li M, Zhang HK and Liang TB: Management strategy for spontaneous isolated dissection of the superior mesenteric artery based on morphologic classification. J Vasc Surg 59: 165-172, 2014.

17. Li T, Zhao S, Li J, Huang Z, Luo C and Yang L: Value of multi-detector CT in detection of isolated spontaneous superior mesenteric artery dissection. Chin Med Sci J 32: 28-33, 2017.

18. Yun WS, Kim YW, Park KB, Cho SK, Do YS, Lee KB, Kim DI and Kim DK: Clinical and angiographic follow-up of spontaneous isolated superior mesenteric artery dissection. Eur J Vasc Endovasc Surg 37: 572-577, 2009.

19. Jia Z, Tu J and Jiang G: The classification and management strategy of spontaneous isolated superior mesenteric artery dissection. Korean Circ J 47: 425-431, 2017.

20. Li S, Gu X, Jiang G and Tian F: Comment on 'The value of a new image classification system for planning treatment and prognosis of spontaneous isolated superior mesenteric artery dissection'. Vascular 23: 558, 2015.
21. Kimura Y, Kato T, Nagao K, Izumi T, Haruna T, Ueyama K, Inada $\mathrm{T}$ and Inoko $\mathrm{M}$ : Outcomes and radiographic findings of isolated spontaneous superior mesenteric artery dissection. Eur J Vasc Endovasc Surg 53: 276-281, 2017.

22. Tomita K, Obara H, Sekimoto Y, Matsubara K, Watada S, Fujimura N, Shibutani S, Nagasaki K, Hayashi S, Harada H, et al: Evolution of computed tomographic characteristics of spontaneous isolated superior mesenteric artery dissection during conservative management. Circ J 80: 1452-1459, 2016.

23. Nath A, Yewale S and Kousha M: Spontaneous isolated superior mesenteric artery dissection. Case Rep Gastroenterol 10: 775-780, 2016.

24. El-Zein RS, Sobecki J, Greenberg R, Keleher M and Palma RA: A spontaneous isolated superior mesenteric artery dissection associated with cocaine abuse: A pathomechanistic association. Case Rep Vasc Med 2020: 2514687, 2020.

25. Park YJ, Park CW, Park KB, Roh YN, Kim DI and Kim YW: Inference from clinical and fluid dynamic studies about underlying cause of spontaneous isolated superior mesenteric artery dissection. J Vasc Surg 53: 80-86, 2011.

26. Mindelzun RE, Jeffrey RB Jr, Lane MJ and Silverman PM: The misty mesentery on CT: Differential diagnosis. AJR Am J Roentgenol 167: 61-65, 1996.

27. Filippone A, Cianci R, Di Fabio F and Storto ML: Misty mesentery: A pictorial review of multidetector-row CT findings. Radiol Med 116: 351-365, 2011

28. Okino Y, Kiyosue H, Mori H, Komatsu E, Matsumoto S, Yamada Y, Suzuki K and Tomonari K: Root of the small-bowel mesentery: Correlative anatomy and CT features of pathologic conditions. Radiographics 21: 1475-1490, 2001

29. Antonopoulos AS, Sanna F, Sabharwal N, Thomas S, Oikonomou EK, Herdman L, Margaritis M, Shirodaria C, Kampoli AM, Akoumianakis I, et al: Detecting human coronary inflammation by imaging perivascular fat. Sci Transl Med 9: eaal2658, 2017

30. Furukawa $\mathrm{H}$ and Moriyama N: Spontaneous dissection of the superior mesenteric artery diagnosed on multidetector helical CT. J Comput Assist Tomogr 26: 143-144, 2002.

31. Takaoka M, Suzuki H, Shioda S, Sekikawa K, Saito Y, Nagai R and Sata M: Endovascular injury induces rapid phenotypic changes in perivascular adipose. Arterioscler Thromb Vasc Biol 30: 1576-1582, 2010.

32. Thanvi B, Munshi SK, Dawson SL and Robinson TG: Carotid and vertebral artery dissection syndromes. Postgrad Med J 81: 383-388, 2005

33. Park KW, Park JS, Hwang SC, Im SB, Shin WH and Kim BT: Vertebral artery dissection: Natural history, clinical features and therapeutic considerations. J Korean Neurosurg Soc 44: 109-115, 2008.

34. Vela D, Buja LM, Madjid M, Burke A, Naghavi M, Willerson JT, Casscells SW and Litovsky S: The role of periadventitial fat in atherosclerosis. Arch Pathol Lab Med 131: 481-487, 2007.

35. Xiong J, Wu Z, Guo W, Liu X, Wang L, Zhang H, Jia X and Ma X: The value of a new image classification system for planning treatment and prognosis of spontaneous isolated superior mesenteric artery dissection. Vascular 23: 504-512, 2015. 